\title{
Process parameters optimization of magnesium alloy quasi-vacuum casting using X-ray image defect inspection
}

\author{
S.-J. Huang, C.-C. Lin*, F.-J. Shiou, J.-Y. Huang \\ Department of Mechanical Engineering, National Taiwan University of Science and Technology, \\ 43, Sec. 4, Keelung Rd. 106, Taiwan, R.O.C.
}

Received 4 August 2016, received in revised form 23 November 2016, accepted 17 February 2017

\begin{abstract}
This study investigated the influence of vacuum casting parameters on defects in magnesium alloy as well as changes in the mechanical properties of these alloys and microstructural evolution under the effects of annealing. We used the Taguchi method in conjunction with an X-ray image detector to determine the percentage of area affected by internal defects, the results of which were verified experimentally. X-ray images of magnesium alloy specimens were filtered, binarized, and edge enhanced. The calculated area of internal defects was then used to optimize process parameters according to multiple performance indicators. Based on the average signal-to-noise ratio, Batch $1\left(720^{\circ} \mathrm{C}, 30\right.$ torr, $2 \mathrm{~h}$, steel, and $120 \mathrm{~min}$ of argon gas injection) and Batch $8\left(780^{\circ} \mathrm{C}, 30\right.$ torr, $1 \mathrm{~h}$, steel, and $120 \mathrm{~min}$ of argon gas injection) were both deemed optimal. The uniformity in the percentage of internal defect area observed in samples from Batch 8 is an indication of stability.
\end{abstract}

K e y w or d s: X-ray image inspection, magnesium alloy casting, defect

\section{Introduction}

Computer vision methodologies based on X-rays are used to characterize casting and welding and facilitate the inspection of baggage and cargo [1]. Marrow et al. [2] applied digital volume correlation to $\mathrm{X}$-ray computed micro-tomography datasets to measure three-dimensional crack opening displacement and thereby identify the crack opening modes in magnesium alloy. Huang et al. [3] studied the mechanical properties of $\mathrm{Mg}$ metal matrix composites (MMCs) with alumina dispersed nanoparticles. They explored the casting of $\mathrm{AM} 60 / \mathrm{AL}_{2} \mathrm{O}_{3 \mathrm{p}} \mathrm{MMCs}$, the strengthening of bar stock manufactured using an equal-channel angular pressing, and the binding ability and tribological behavior of base materials. Lin et al. [4] investigated the microstructural evolution and mechanical properties of AZ31B magnesium alloy. Jacob et al. [5] proposed the use of Taguchi method to optimize the control variables to reduce casting defects. A numerical simulation is a powerful tool for solving linear as well as nonlinear problems. Benezeth et al. [6] developed a method for evaluation of foreground detection algorithms objectively. Dubroca et al. [7] developed an algorithm for the classification of spectral data, wherein an acoustic signal is broadcast if a threat is detected. Latos et al. [8] introduced a novel method for the testing of automated X-ray inspection systems. Unfortunately, the methods currently used by many engineers and inspection system manufacturers do not test grayscale or positioning stability in relation to changes that occur over time. Pei et al. [9] used energy minimization to label pixels associated with every camera view to determine whether they belong to an occlusion. Chukalina et al. [10] demonstrated that the estimators of the coefficients of linear attenuation in the media are in good agreement with the requirements of tomography devices used in industry, laboratories, and medical facilities. Shui and Zhang [11] proposed a corner detector and classifier using anisotropic directional derivative (ANDD) representations.

Bay et al. [12] presented a novel scale- and rotation-invariant detector and descriptor referred to as Speeded-Up Robust Features. It has been widely used in the field of computer vision. 
Table 1. Elements and their corresponding atomic numbers: $<10,10-18$, and $>18$

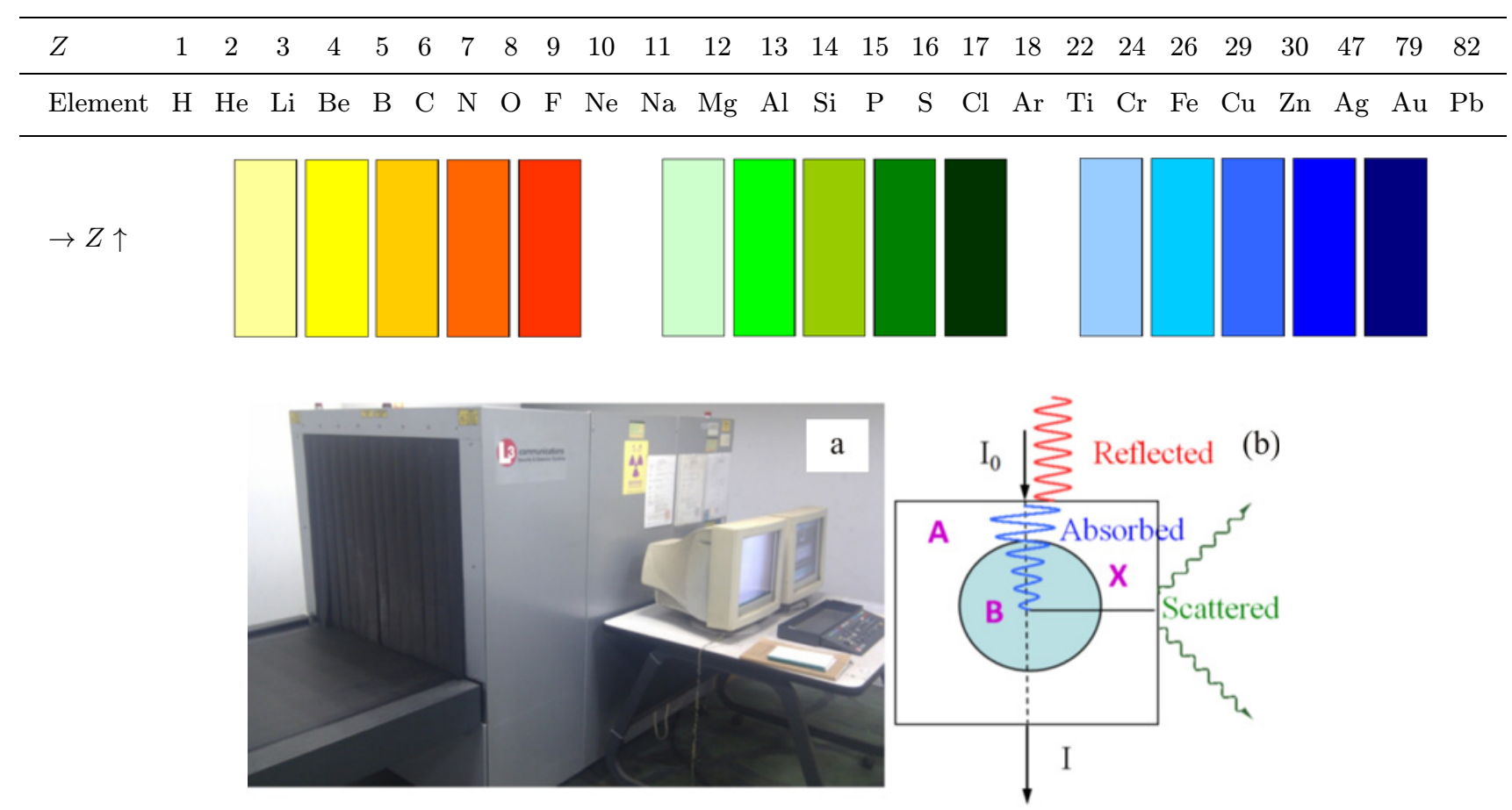

Fig. 1. Object undergoing examination (a) and X-ray contrast (b).

Micro defects can grow into micro cracks, which expand and converge through repeated stress into macroscopic cracks, leading to fatigue damage and fracturing in the structure [13-14]. X-ray computed tomography has been used to determine the pore fraction and pore distribution in $\mathrm{Al}$ and $\mathrm{Mg}$ alloy castings [1-2, 15-16]. This method can detect defects; however, it cannot be used to measure the percentage of internal defect area (PIDA). In this study, we used X-ray image detection to determine the PIDA. This is then used as a criterion for the Taguchi method, which aims to optimize the quasi-vacuum casting of $\mathrm{Mg}$ alloy.

\section{Principles of non-destructive X-ray examination}

X-ray imaging can be used to detect interatomic distance, characterize crystal structures, and identify internal micro defects in materials. This study used non-destructive X-ray fluoroscopy to examine the PIDA of magnesium alloy obtained using vacuum casting.

\subsection{Fundamental principles of X-ray image inspection}

X-rays that come into contact with any form of matter are partly transmitted and partly absorbed. Figure 1 and Eqs. (1) and (2) show that the emitted $\mathrm{X}$-rays are less intense than the incident X-rays $\left(I_{0}\right)$, as reported by Cullity and Stock [17]:

$$
\begin{gathered}
I=I_{0} \mathrm{e}^{-\tau d}, \\
\tau=\alpha Z^{3} \rho \lambda^{3},
\end{gathered}
$$

where $\tau$ is the absorption coefficient, $\alpha$ is a constant, and $\tau / \rho$ is the mass absorption coefficient. The difference $\left(I_{0}-I_{1}\right)$ is the amount of X-rays absorbed by the object under the effects of scattering. Absorption is proportional to the thickness $(d)$, density $(\rho)$, atomic number $(Z)$, and wavelength $(\lambda)$ of the specimen.

Using the B field of the X-ray photons with intensity $I_{\mathrm{B}}$, and the A field with $I_{\mathrm{A}}, \mathrm{Eq}$. (3) is used to calculate the $\mathrm{X}$-ray contrast as follows:

$$
C_{\mathrm{AB}}=\frac{I_{\mathrm{A}}-I_{\mathrm{B}}}{I_{\mathrm{A}}} \times 100 \text { and } \bar{C}_{\mathrm{A}, \mathrm{B}}=\frac{I_{\mathrm{A}}-I_{\mathrm{B}}}{I_{\mathrm{A}}+I_{\mathrm{B}}}
$$

where $I_{\mathrm{A}}$ and $I_{\mathrm{B}}$ are the intensities of the X-rays of objects $A$ and $B$, respectively. Field $B$ is the target and Field $\mathrm{A}$ is the remaining region.

Equation (4) expresses the concentration and contrast of X-rays, as reported by Cullity and Stock [17]:

$$
-\log _{10} \frac{I}{I_{0}}=\log _{10} \frac{I_{0}}{I}
$$

where $I_{0}$ is the intensity of incident X-rays, and $I$ is the intensity of through X-rays. 


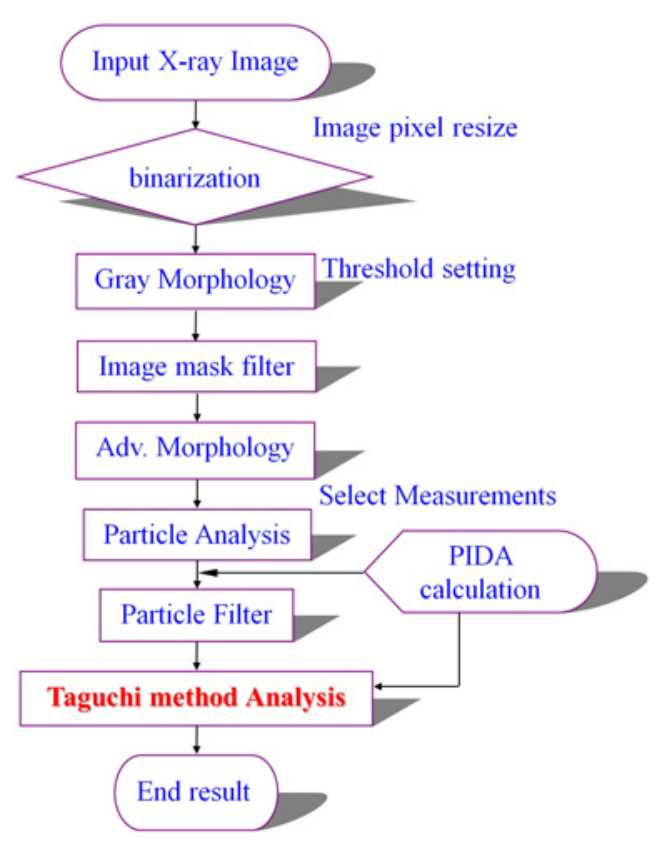

Fig. 2. Flowchart for processing X-ray images.

\subsection{Fundamental principles of $X$-ray detection using a spectrometer}

In this study, a Perkin Elmer facility-model X-ray baggage inspection machine (shown in Fig. 1) was used for X-ray detection. The test parameters used in the experiment were $160 \mathrm{kVp}, 1.0 \mathrm{~mA}$, background radiation dose of $0.09-0.15$, and a continuous model. The specifications for the rendering of color are described in the following. The darkness of the color on the monitor increases with an increase in atomic number $Z$, as shown in Table 1 .

\subsubsection{Orange: First category}

Lighter elements with an atomic number of less than 10 (such as carbon, nitrogen, or oxygen), appear in the orange group. All organic materials fall into this category.

\subsubsection{Green: Second category}

Medium-weight elements, with atomic numbers of 10-18, appear in the green group. Some are light- and medium-weight, depending on the quality of the mixture. X-ray radiation can penetrate organic as well as inorganic materials.

\subsubsection{Blue: Third category}

Elements with atomic numbers exceeding 18 appear in the blue group. Some metals used in the fabrication of knives and guns fall into this category.
Table 2. Chemical composition (wt.\%) of magnesium alloy AZ31

\begin{tabular}{ccccccc}
\hline $\mathrm{Al}$ & $\mathrm{Zn}$ & $\mathrm{Mn}$ & $\mathrm{Si}$ & $\mathrm{Ni}$ & $\mathrm{Fe}$ & $\mathrm{Mg}$ \\
\hline 3.01 & 0.9 & 0.5 & 0.04 & 0.005 & 0.005 & balance \\
\hline
\end{tabular}

Table 3. Levels of control factors

\begin{tabular}{lcc}
\hline Parameter name & First level & Second level \\
\hline Heating temperature $T\left({ }^{\circ} \mathrm{C}\right)$ & 720 & 780 \\
Degree of vacuum $V$ (torr) & 30 & 50 \\
Holding temperature time $t(\mathrm{~h})$ & 2 & 1 \\
Heating medium $m$ & Steel ball & $\mathrm{Al}_{2} \mathrm{O}_{3}$ \\
Inert gas $G$ & Ar 120 min & $\mathrm{Ar} 60$ min \\
\hline
\end{tabular}

The computer image processing technology in Fig. 2 was used to develop a binarization algorithm for the processing of X-ray images. The X-ray images are RGB (32 bit); however, the threshold step supports only binary, 8 bit, 16 bit, and floating-point image types. The threshold was set to grayscale images using a specific range of pixel values. The gray morphology was then used to modify the shape of objects in an image. Open and Close algorithms were used to process the images based on the Erode-and-Dilate Method. Image masking was then used to build a mask from an entire image or a region selected from an image. The advanced morphology was then used to perform high-level operations on flaws in binary images and remove small objects (Iterations: 10). Particle analysis was used to display measurement results for selected particle measurements performed on the image (Select Measurements: area of the particle). Find the internal defect distribution with annotations. Every PIDA count with the algorithm and help of software. Finally, a particle filter was used to remove or retain particles by the filter criteria (Parameter Range: Minimum range: 2000, Maximum range: 2500. Action: Keep).

\section{Experiment design}

The material used for X-ray inspection was magnesium alloy AZ31. Table 2 lists the chemical composition of the AZ31, which was manufactured under vacuum casting. Table 3 lists the levels of the control factors used as experiment parameters. The control conditions in this study were as follows: heating temperature $(T)$, the degree of vacuum $(V)$, holding temperature time $(t)$, heating medium $(m)$, and inert gas $(G)$. In the first and second levels, $T$ values were 720 and $780{ }^{\circ} \mathrm{C}, V$ values were 30 and 50 torr, $t$ was 2 and $1 \mathrm{~h}, m$ involved a steel ball and $\mathrm{Al}_{2} \mathrm{O}_{3}$, and $G$ 


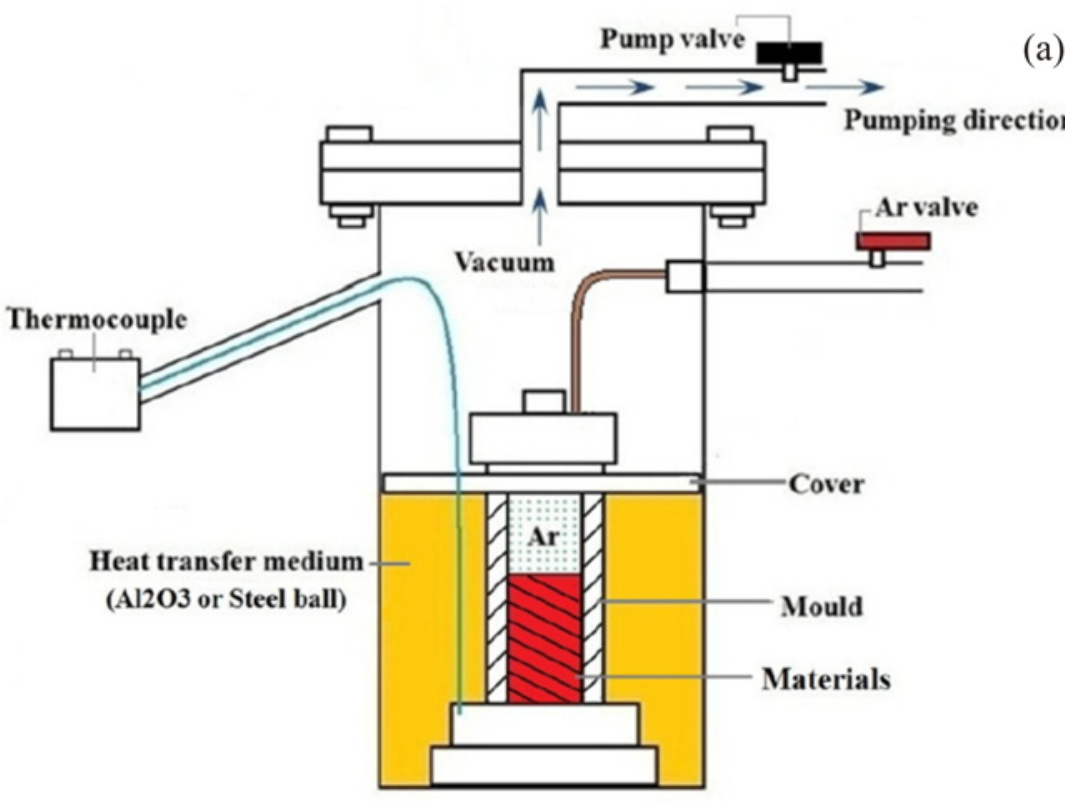

(a)

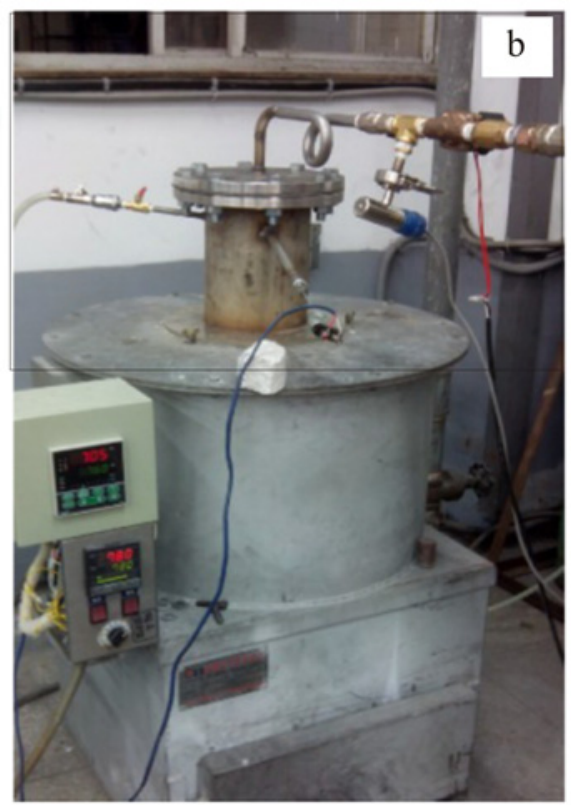

Fig. 3a,b. Vacuum casting equipment for manufacturing of magnesium alloy materials.

Table 4. Vacuum casting process

\begin{tabular}{lcl}
\hline & Temperature & Process \\
\hline Stage 1 & Room temperature $\left({ }^{\circ} \mathrm{C}\right)$ & The entire mold is vacated using a vacuum pump. \\
\hline Stage 2 & 200 & $\begin{array}{l}\text { With the vacuum pump operating, the external mold is vacated, and trace } \\
\text { argon is injected into the mold. }\end{array}$ \\
\hline Stage 3 & 700 & With the vacuum pump operating, argon gas is injected into the mold. \\
\hline Stage 4 & 780 & With the vacuum pump operating, argon gas is injected into the mold. \\
\hline Stage 5 & $\begin{array}{l}\text { The mold is vacated one last time, during which the constant temperature } \\
\text { is maintained for } 1 \text { h to enable the magnesium alloy to diffuse and melt } \\
\text { fully. }\end{array}$ \\
\hline Stage 6 & $\begin{array}{l}\text { The high-temperature sintering furnace starts cooling. The vacuum pump } \\
\text { continues operating (uninterrupted) to enable the mold to cool down slowly. }\end{array}$ \\
\hline Stage 7 & \begin{tabular}{l} 
The vacuum pump continues operating. \\
\hline Stage 8
\end{tabular} & $\begin{array}{l}\text { The vacuum pump is turned off, the ambient air is purged using argon gas, } \\
\text { and the sample is allowed to stand for cooling. }\end{array}$ \\
\hline
\end{tabular}

values were 120 and 60 min, respectively. The Taguchi method was used to identify the optimal process control factors and their influence on the defect area of the magnesium alloy material. The defect area was examined using X-ray image inspection in conjunction with image processing to optimize process quality.

Conventional magnesium alloy is manufactured using die casting or precision casting. If the mold cavity is not vacated properly, then small amounts of gas or vapor may be absorbed during the packing or cooling process. Image processing was used to estimate the size and density of the defect areas. This study developed a special vacuum casting mold using a small customized mold to reduce costs and enhance quality.

Figure 3 presents the vacuum casting equipment used for the manufacture of the magnesium alloy materials, wherein aluminum oxide or steel balls were used as the heat transfer media. The vacuum casting 
Table 5. Orthogonal array $\mathrm{L}_{8}\left(2^{7}\right)$ for parameter arrangement based on the Taguchi method

\begin{tabular}{|c|c|c|c|c|c|c|c|c|}
\hline \multirow{3}{*}{ Batch no. } & & \multirow{2}{*}{$\begin{array}{c}T \\
\left({ }^{\circ} \mathrm{C}\right)\end{array}$} & \multirow{2}{*}{$\begin{array}{c}V \\
\text { (torr) }\end{array}$} & \multirow{2}{*}{$\begin{array}{c}t \\
(\mathrm{~h})\end{array}$} & \multirow{2}{*}{$m$} & \multirow{2}{*}{$\begin{array}{c}G \\
(\min )\end{array}$} & \multirow{2}{*}{$\frac{\text { Defect }}{\text { Defect area }}$} & \multirow{2}{*}{ Note } \\
\hline & & & & & & & & \\
\hline & 1 & 2 & 3 & 4 & 5 & 6 & Pixel area & no. \\
\hline \multirow[t]{2}{*}{1} & 1 & 720 & 30 & 2 & Steel & 120 & 106 & 15 \\
\hline & & & & & & & 344 & 16 \\
\hline \multirow[t]{2}{*}{2} & 1 & 720 & 30 & 1 & $\mathrm{Al}_{2} \mathrm{O}_{3}$ & 60 & 518 & 1 \\
\hline & & & & & & & 271 & 2 \\
\hline \multirow[t]{2}{*}{3} & 1 & 780 & 50 & 2 & Steel & 60 & 480 & 13 \\
\hline & & & & & & & 129 & 14 \\
\hline \multirow[t]{2}{*}{4} & 1 & 780 & 50 & 1 & $\mathrm{Al}_{2} \mathrm{O}_{3}$ & 120 & 1080 & 3 \\
\hline & & & & & & & 516 & 4 \\
\hline \multirow[t]{2}{*}{5} & 2 & 720 & 50 & 2 & $\mathrm{Al}_{2} \mathrm{O}_{3}$ & 120 & 1005 & 5 \\
\hline & & & & & & & 1106 & 6 \\
\hline \multirow[t]{2}{*}{6} & 2 & 720 & 50 & 1 & Steel & 60 & 479 & 9 \\
\hline & & & & & & & 643 & 10 \\
\hline \multirow[t]{2}{*}{7} & 2 & 780 & 30 & 2 & $\mathrm{Al}_{2} \mathrm{O}_{3}$ & 60 & 487 & 7 \\
\hline & & & & & & & 761 & 8 \\
\hline \multirow[t]{2}{*}{8} & 2 & 780 & 30 & 1 & Steel & 120 & 346 & 11 \\
\hline & & & & & & & 385 & 12 \\
\hline
\end{tabular}

Ta bl e $6 . \mathrm{L}_{8}\left(2^{7}\right)$ experiment based on the Taguchi method

\begin{tabular}{|c|c|c|c|c|c|c|c|c|c|c|}
\hline \multirow{3}{*}{ Batch no. } & & \multirow{2}{*}{$\begin{array}{c}T \\
\left({ }^{\circ} \mathrm{C}\right)\end{array}$} & \multirow{2}{*}{$\begin{array}{c}V \\
\text { (torr) }\end{array}$} & \multirow{2}{*}{$\begin{array}{c}t \\
(\mathrm{~h})\end{array}$} & \multirow{2}{*}{$m$} & \multirow{2}{*}{$\begin{array}{c}G \\
(\min )\end{array}$} & & & \multirow{2}{*}{$\begin{array}{c}S / N \text { ratio } \\
\quad(\mathrm{db})\end{array}$} & \multirow{2}{*}{ Mean } \\
\hline & & & & & & & \multicolumn{2}{|c|}{$\begin{array}{c}\text { Defect } \\
\text { Defect area }(\%)\end{array}$} & & \\
\hline & 1 & 2 & 3 & 4 & 5 & 6 & \multicolumn{2}{|c|}{ Pixel area } & & \\
\hline 1 & 1 & 720 & 30 & 2 & Steel & 120 & 0.2808 & 0.7339 & 5.1042 & 0.5074 \\
\hline 2 & 1 & 720 & 30 & 1 & $\mathrm{Al}_{2} \mathrm{O}_{3}$ & 60 & 1.1959 & 0.5856 & 0.5230 & 0.8908 \\
\hline 3 & 1 & 780 & 50 & 2 & Steel & 60 & 0.9702 & 0.2458 & 3.0029 & 0.6080 \\
\hline 4 & 1 & 780 & 50 & 1 & $\mathrm{Al}_{2} \mathrm{O}_{3}$ & 120 & 1.9945 & 0.9599 & -3.8912 & 1.4772 \\
\hline 5 & 2 & 720 & 50 & 2 & $\mathrm{Al}_{2} \mathrm{O}_{3}$ & 120 & 1.8138 & 2.286 & -6.2919 & 2.0499 \\
\hline 6 & 2 & 720 & 50 & 1 & Steel & 60 & 1.2351 & 1.4344 & -2.5321 & 1.3348 \\
\hline 7 & 2 & 780 & 30 & 2 & $\mathrm{Al}_{2} \mathrm{O}_{3}$ & 60 & 0.1071 & 1.8738 & -2.4583 & 0.9905 \\
\hline 8 & 2 & 780 & 30 & 1 & Steel & 120 & 0.6054 & 0.6222 & 4.2386 & 0.6138 \\
\hline
\end{tabular}

process was as follows: the vacuum mold was placed in a high-temperature sintering furnace and heated to a set temperature, and then vacated using a vacuum pump to fully remove residual gas and steam from the mold. Argon was injected into the mold to enhance the conduction and convection of heat. The temperature was measured using a thermocouple. When the temperature inside the mold reached $700^{\circ} \mathrm{C}$, the mold was fully evacuated until the magnesium alloy solidified (Table 4).

The specimens with a small defect area (Table 5) were selected according to the concept of the-smaller-the-better using the Taguchi parameter design method. The optimum combination of parameters was verified experimentally.

The hardness of the specimens was tested using a micro Vickers hardness tester under a load of 300 gf $(2.943 \mathrm{~N})$, and loading time of $15 \mathrm{~s}$, using VHPro Express for calculations. The tester makes a rhombic indentation using a diamond indenter, and the average length of the diagonal of the indentation is substituted into Eq. (4) to determine the Vickers hardness. The specimens were tested at 15 points, and the mean of the results was obtained as follows:

$$
\mathrm{HV}=0.102 \times \frac{F}{S}=0.102 \times \frac{2 F \sin \frac{\alpha}{2}}{d^{2}}
$$

where $F$ is load, $S$ is the surface area of indentation, $\alpha$ included an angle of the indenter, and $d$ is the average length of indentation diagonal. 


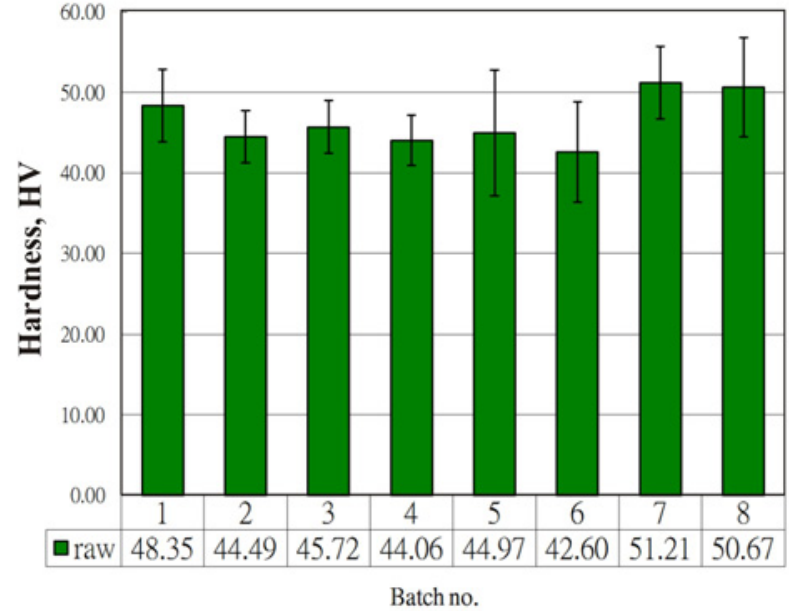

Fig. 4. Mean Vickers hardness of AZ31 magnesium alloy specimens manufactured using quasi-vacuum casting using parameters derived by the Taguchi method.

\section{Results of Taguchi method}

The micro defects are internal; therefore, the defect area and density cannot be examined with the naked eye, i.e., they must be analyzed using computer image processing, the results of which are shown in Fig. 5.

The PIDA values are shown in Table 6. The crystalline structure and morphology of the magnesium
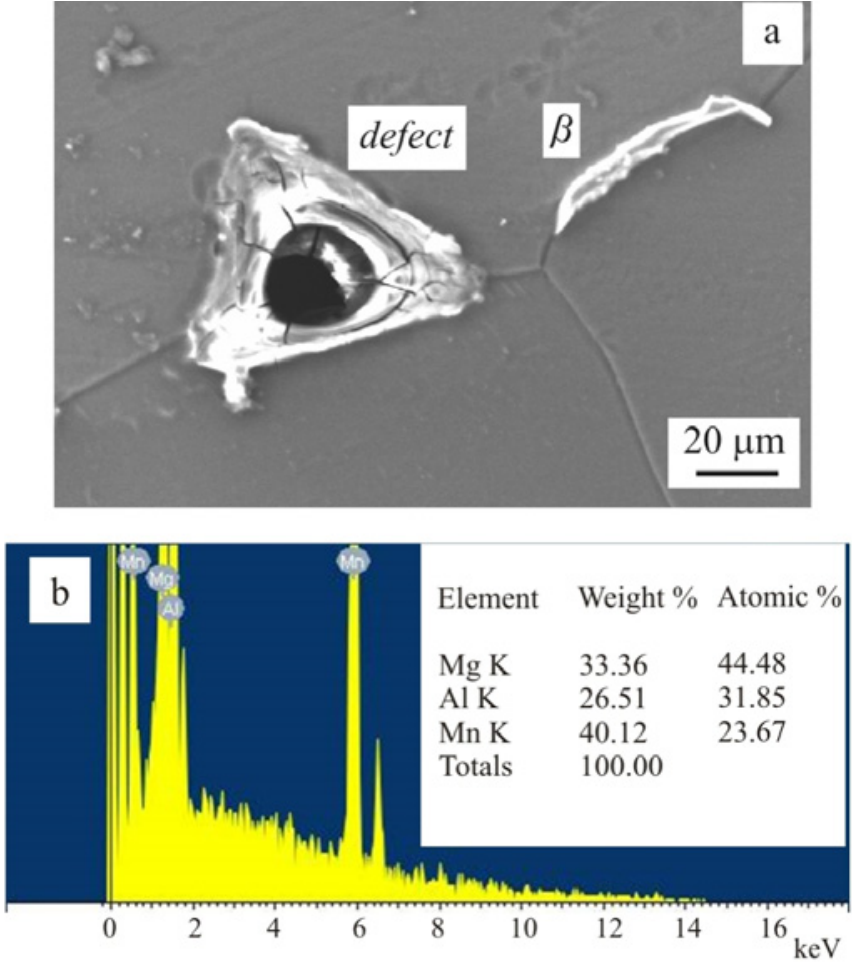

Fig. 6. Crystalline structure and morphology of the defect and $\beta$-phase as characterized using (a) scanning electron microscopy and (b) energy-dispersive X-ray spectroscopy.

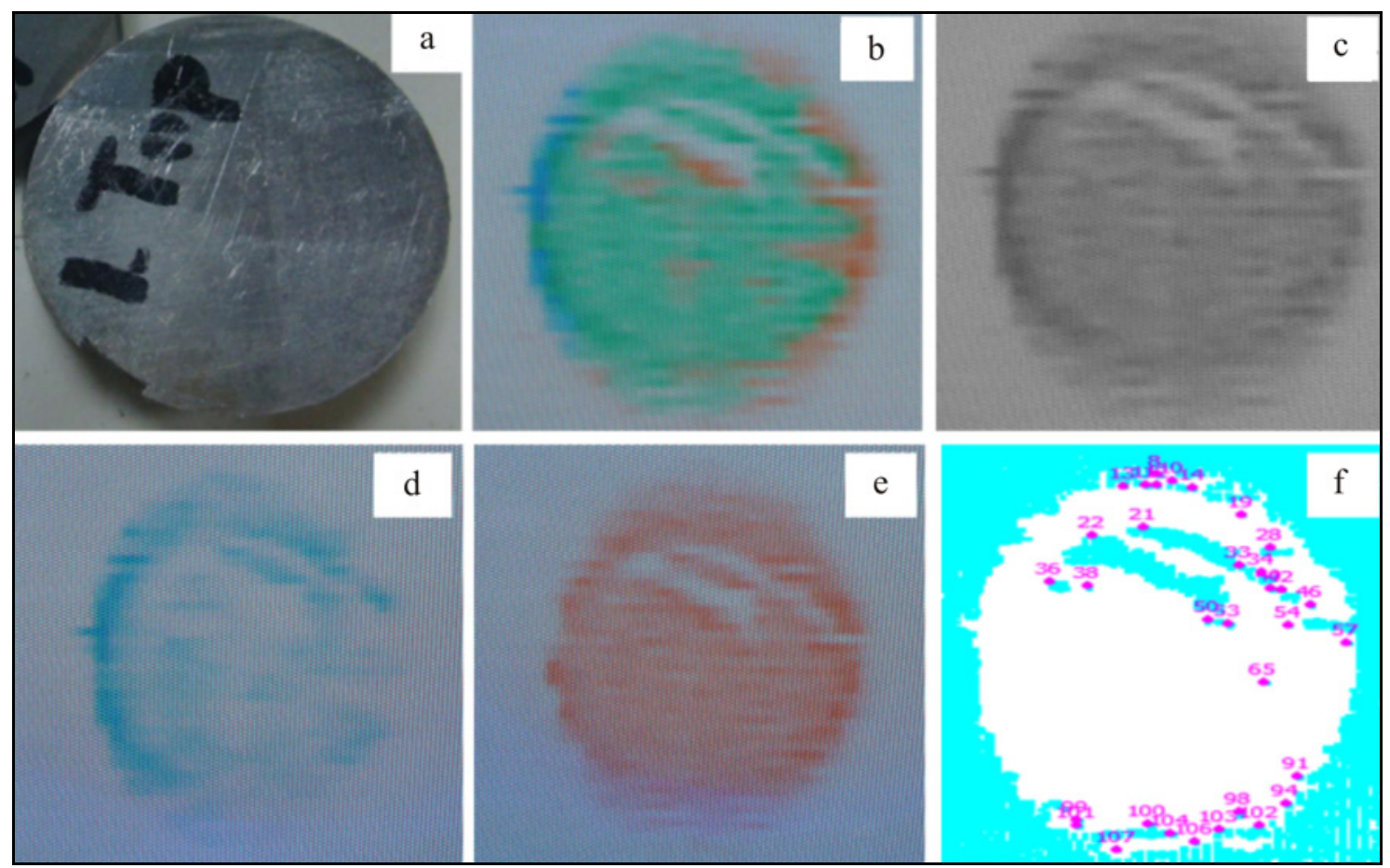

Fig. 5. X-ray images of magnesium alloy specimen: (a) test specimen; (b) original X-ray image; (c) grayscale image; (d) image that excludes inorganics and retains organics; (e) image that excludes organics and retains inorganics; and (f) image with annotations of the internal defect distribution. 


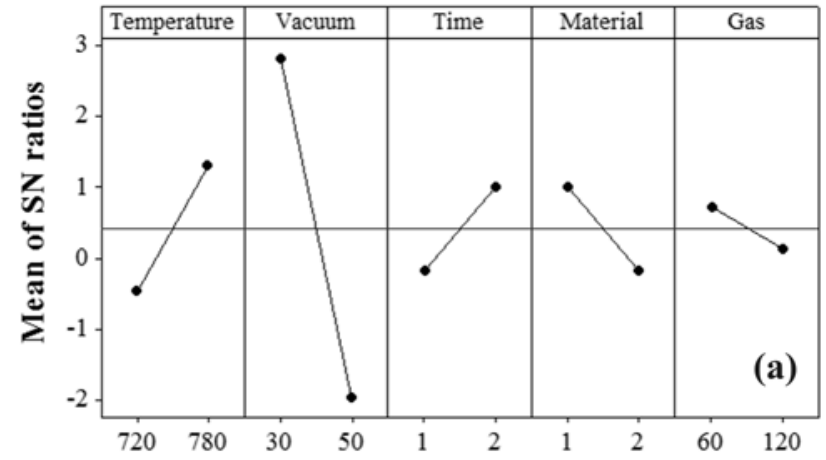

Signal-to-noise: Smaller is better

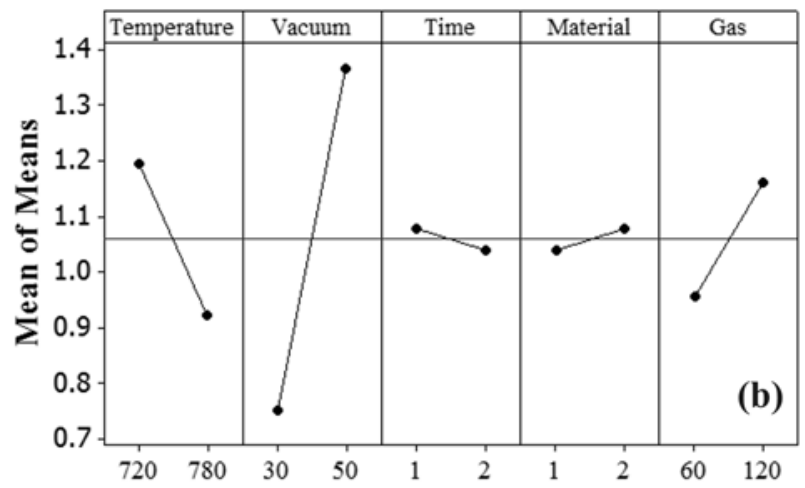

Fig. 7. Reaction graphs of (a) $S / N$ ratios and (b) average values.

alloy were characterized using an X-ray energy dispersive spectrometer and scanning electron microscope, respectively, as shown in Fig. 6. Spectrum 2 indicates the micro defects within the magnesium alloy, ranging in size from 10 to $20 \mu \mathrm{m}$.

Figure 7 presents reaction graphs of the signal-to-noise $(S / N)$ ratios presented with the effects of each factor, commonly referred to as the main effect. A high
$S / N$ ratio is superior to a low $S / N$ ratio. A considerable difference was observed between the levels of temperature $(T)$ and vacuum $(V)$. The average values in the reaction graphs revealed considerable differences in the factor levels of $V$, which indicates that this parameter is highly sensitive to process reaction and PIDA changes, making it the key variable influencing the process. Tables 7 and 8 present the delta values of all levels ranked according to size. These results indicate that the reaction values were most substantially influenced by $V$, followed by $T$ and $G$. The average values of the other two factor levels did not differ significantly.

The design of the Taguchi orthogonal array was $\mathrm{L}_{8}\left(2^{5}\right)$, with eight rows and five "2 level" columns. The Taguchi trials involved five control factors: defect area versus $T, V, t, m$, and $G$.

Our results revealed that the design was not orthogonal and the physical factors were highly correlated with the other terms. The response for $S / N$ ratios and the average for different factor levels are presented in Tables 7 and 8, using the quality loss function (QLF) for the smaller-the-better. Optimal parameters and their levels were determined according to experimental outputs. Table 6 presents the optimal combination of control factors based on the highest average $S / N$ ratios. Figure 7 presents the main effect plot against the $S / N$ ratio. High $S / N$ ratios were assembled into an optimal combination as follows: $T_{2}$, $V_{1}, t_{2}, m_{1}$, and $G_{2}$.

$\mathrm{X}$-ray diffraction analysis was conducted in crosssections in the middle section of the AZ31 magnesium alloy casting. The results of the Vickers hardness test in Fig. 4 indicate that Taguchi batch no.1 (HV 48.35) and batch no.8 (HV 50.67) have higher hardness values than the other specimens. Furthermore, the frequency spectrum in Fig. 8 shows that the casting was composed primarily of a base phase

Table 7. $S / N$ ratios

\begin{tabular}{cccccc}
\hline Level & Temperature & Vacuum & Time & Media & Gas \\
\hline 1 & -0.7992 & 1.8519 & -0.4154 & -0.1608 & -0.3662 \\
2 & 0.2230 & -2.4281 & -0.1608 & -0.4154 & -0.2100 \\
Delta & 1.0222 & 4.2799 & 0.2547 & 0.2547 & 0.1561 \\
Rank & 2 & 1 & 3.5 & 3.5 & 5 \\
\hline
\end{tabular}

Table 8. Averages of various factor levels

\begin{tabular}{cccccc}
\hline Level & Temperature & Vacuum & Time & Media & Gas \\
\hline 1 & 1.1957 & 0.7506 & 1.0791 & 1.0389 & 0.9560 \\
2 & 0.9224 & 1.3675 & 1.0389 & 1.0791 & 1.1621 \\
Delta & 0.2733 & 0.6169 & 0.0402 & 0.0402 & 0.2061 \\
Rank & 2 & 1 & 4.5 & 4.5 & 3 \\
\hline
\end{tabular}



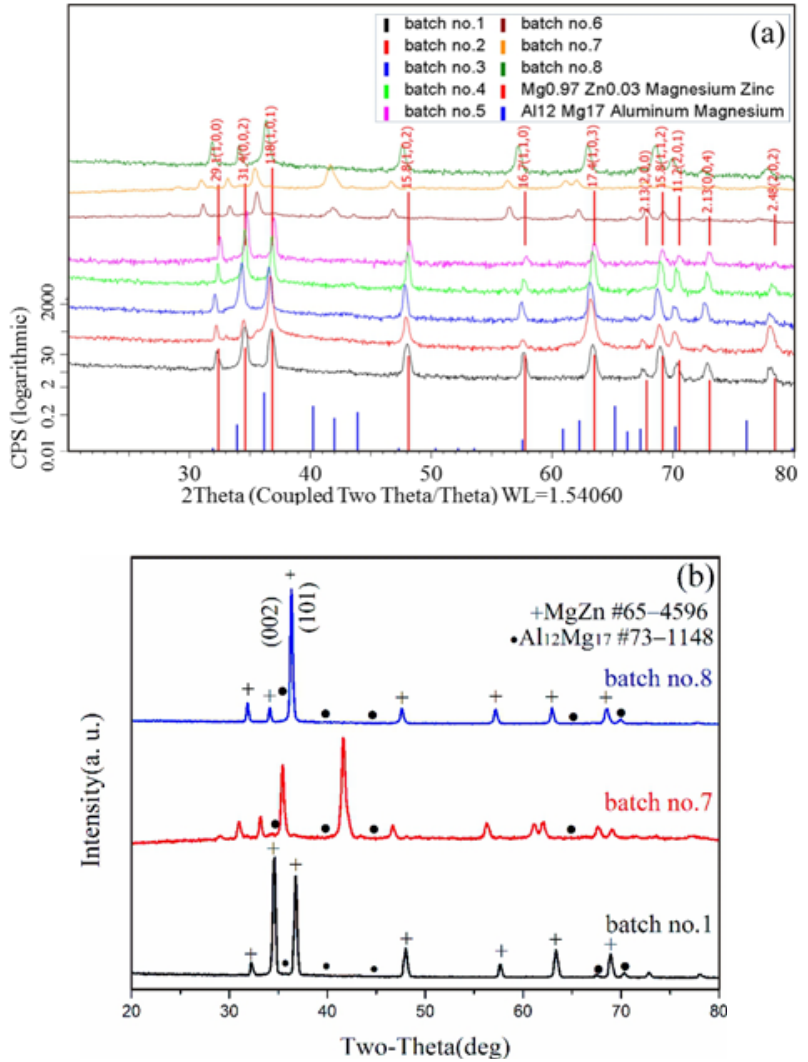

Fig. 8. XRD patterns of AZ31 alloys: (a) batch no.1-batch no. 8 and (b) batch no.1, 7 and 8 by $\mathrm{L}_{8}\left(2^{7}\right)$ in Taguchi experiments.

and $\beta$-phase $\left(\mathrm{Mg}_{17} \mathrm{Al}_{12}\right)$ of magnesium. The $\beta$-phase content was higher in batches no. 1,7 , and 8 , which made those specimens harder. The peak values derived from X-ray diffraction indicate the crystallinity of the planes. In quasi-vacuum casting, the intensities of the diffraction peaks did not present significant differences. The material of the casting presented a preferred orientation, which means that the grains in the crystals arranged themselves in specific directions. The results in Fig. 8 indicate optimal growth in batches no.1 and 8, where (002) and (101) present higher peak values indicative of superior crystallinity. The results of X-ray imaging and Taguchi analysis indicate that superior crystallinity led to smaller PIDA values in batches no. 1 and 8 , thereby was done by optimizing the control variables or process parameters.

\section{Conclusions}

Our goal in this experiment was to minimize the PIDA to improve the quality of the magnesium alloy quasi-vacuum casting. An X-ray image detector was used for the non-destructive investigation of the magnesium alloy, and PIDA values were calculated using a novel image processing method developed in this study. Our results indicate that $T$ and $V$ were the factors with the greatest influence. For image processing, the X-ray images of the magnesium alloy specimens were filtered, binarized, and edge-enhanced, and the PIDA was calculated using a morphological algorithm. In conclusion, the base phase and $\beta$-phase of magnesium in batches no. 1, 7, and 8 produced specimens with higher hardness values. Superior crystallinity led to smaller PIDA values in batches no. 1 and 8. Taguchi analysis led to the following optimal combination of parameters: $T_{2}\left(780^{\circ} \mathrm{C}\right), V_{1}$ (30 torr), $t_{2}(1 \mathrm{~h}), m_{1}$ (steel ball media), and $G_{2}$ (Ar gas $\left.60 \mathrm{~min}\right)$.

\section{References}

[1] Mery, D.: Insight, 56, 2014, p. 147. doi:10.1784/insi.2014.56.3.147

[2] Marrow, T. J., Mostafavi, M., Hashimoto, T., Thompson, G. E.: Int. J. Fatigue, 66, 2014, p. 183. doi:10.1016/j.ijfatigue.2014.04.003

[3] Huang, S. J., Lin, P. C., Ballóková, B., Hvizdoš, P., Besterci, M.: Kovove Mater., 52, 2014, p. 313. doi:10.4149/km_2014_5_313

[4] Lin, C. M., Liu, J. J., Tsai, H. L., Cheng, C. M.: J. Chin. Inst. Eng., 34, 2011, p. 1013. doi:10.1080/02533839.2011.618242

[5] Jacob, A. B., Arunkumar, O. N.: Journal of Mechanical and Civil Engineering, 1, 2016, p. 67.

[6] Benezeth, Y., Emile, B., Rosenberger, C.: IEEE Fourth International Conference on Image and Graphics, 2007, p. 661. doi:10.1109/ICIG.2007.68

[7] Dubroca, T., Brown, G., Hummel, R. E.: Opt. Eng., 53, 2014, p. 021112-1. doi: 10.1117/1.OE.53.2.021112

[8] Latos, I., Janóczki, M.: Solder. Surf. Mt. Tech., 23, 2011, p. 91. doi: 10.1108/09540911111120168

[9] Pei, Z., Zhang, Y., Chen, X., Yang, Y. H.: Pattern Recogn., 46, 2013, p. 174. doi: 10.1016/i.patcog.2012.06.014

[10] Chukalina, M. V., Buzmakov, A. V., Nikolaev, D. P., Chulichkov, A. I., Karimov, M. G., Rasulov, G. A., Senin, R. A., Asadchikov, V. E.: Meas. Tech+., 51, 2008, p. 136. doi:10.1007/s11018-008-9015-3

[11] Shui, P. L., Zhang, W. C.: IEEE T. Image Process., 22, 2013, p. 3204. doi: 10.1109/TIP.2013.2259834

[12] Bay, H., Ess, A., Tuytelaars, T., Gool, L. V.: Comput. Vis. Image Und., 110, 2008, p. 346. doi: 10.1016/j.cviu.2007.09.014

[13] Huang, S. J., Lin, Y. C.: J. Adhesion Sci. Technol., 18, 2004, p. 833. doi: 10.1163/156856104840309.

[14] Velgosová, O., Besterci, M., Kováč, L., Kulu, P., Huang, S. J.: Kovove Mater., 49, 2011, p. 361. doi: $10.4149 / \mathrm{km} \_2011 \_5 \_361$

[15] Wan, Q., Zhao, H., Zou, C.: ISIJ International, 54, 2014, p. 511. doi: 10.2355 /isijinternational.54.511

[16] Kan, K., Imura, Y., Morii, H., Kobayashi, K., Minemura, T., Aoki, T.: World Journal of Nuclear Science and Technology, 3, 2013, p. 106. doi:10.4236/winst.2013.33018

[17] Cullity, B. D., Stock, S. R.: Elements of X-ray Diffraction. 3rd Edition. New Jersey, Prentice-Hall 2001. 